\title{
Influence of Fructooligosaccharide on Pharmacokinetics of Isoflavones in Postmenopausal Women
}

\author{
Supanimit Teekachunhatean,, ${ }^{1,2}$ Sujitra Techatoei, ${ }^{1,2}$ Noppamas Rojanasthein, ${ }^{1}$ \\ Maleeya Manorot, ${ }^{1}$ and Chaichan Sangdee ${ }^{1}$ \\ ${ }^{1}$ Department of Pharmacology, Faculty of Medicine, Chiang Mai University, Chiang Mai 50200, Thailand \\ ${ }^{2}$ Center of Thai Traditional and Complementary Medicine, Faculty of Medicine, Chiang Mai University, Chiang Mai 50200, Thailand
}

Correspondence should be addressed to Supanimit Teekachunhatean, steekach@med.cmu.ac.th

Received 13 December 2011; Revised 24 January 2012; Accepted 26 January 2012

Academic Editor: Angelo Antonio Izzo

Copyright () 2012 Supanimit Teekachunhatean et al. This is an open access article distributed under the Creative Commons Attribution License, which permits unrestricted use, distribution, and reproduction in any medium, provided the original work is properly cited.

\begin{abstract}
The objective of the present paper was to determine the influence of fructooligosaccharide (FOS) on the pharmacokinetics of isoflavones in healthy postmenopausal women. The study was a fixed-sequence, two-phase, crossover study. Twelve subjects received a single oral dose of $300 \mathrm{~mL}$ of a soy beverage. Blood samples were collected before the dose and at $0.5,1,2,4,6,8,10,12$, 24 , and $32 \mathrm{~h}$ after the administration of the soy beverage. After a washout period of at least 1 week, subjects were assigned to receive oral doses of FOS, $5 \mathrm{~g}$ each time, twice a day (after breakfast and dinner) for 14 days, followed by a single oral dose of the same soy beverage on the next day. Blood samples were then collected at the same time points mentioned previously. Plasma isoflavone concentrations were determined by HPLC. Continuous oral administrations of FOS followed by a single oral administration of soy beverage caused significant increases in $C_{\max }, \mathrm{AUC}_{0-32}$, and $\mathrm{AUC}_{0-\infty}$ of genistein and $\mathrm{AUC}_{0-32}$ of daidzein, comparing to those obtained following a single oral dose of soy beverage alone. Other pharmacokinetic parameters ( $T_{\max }$ and $t_{1 / 2}$ of both aglycones and $\mathrm{AUC}_{0-\infty}$ of daidzein) between both regimens were not significantly different.
\end{abstract}

\section{Introduction}

Decreased estrogen level demonstrated in postmenopausal women commonly relates to a variety of disorders, for example, hot flushes, osteoporosis, depression, increased body weight, and so forth. As a therapeutic goal, hormone replacement therapy (HRT) has been used for decades as the "gold standard" to treat estrogen deficiency symptoms [1]. Estrogen therapy has been demonstrated to benefit postmenopausal women mostly through the relief of vasomotor symptoms as well as for the prevention and treatment of osteoporosis [2]. However, estrogen therapy has been shown to increase the risk of breast cancer, uterine and endometrial cancer, as well as menstrual bleeding [3]. Although the incidence of endometrial cancer can be avoided by using estrogen plus progestin [4], this combination increases the risks of stroke, pulmonary embolism, and invasive breast cancer [5]. Therefore, both postmenopausal women and physicians remain concerned about the adverse effects of estrogen and are looking for natural products that possess beneficial effects similar to estrogen but have more favorable safety profiles. Indeed, phytoestrogens offer attractive options because they have been demonstrated to decrease the risk of endometrial and breast cancer, avoid the need for progestin, have fewer adverse reactions, and still provide health benefits [6].

Isoflavones represent the most common group of phytoestrogens, and soybeans are the most common source of isoflavones in human food. Isoflavones are structurally similar to estrogen but possess weaker hormonal effects. They bind weakly to the estrogen receptor alpha $(\mathrm{ER} \alpha)$ of the reproductive organs such as the uterus, ovaries, and breast, but bind more strongly to the estrogen receptor beta $(\mathrm{ER} \beta)$ found in the brain, arteries and bone [7], and have 
been associated with beneficial effects in humans, such as the relief of hot flushes, and the prevention of osteoporosis, cardiovascular diseases, and cancers [8].

The unconjugated aglycones (daidzein, genistein, and glycitein) are contained predominantly in fermented soybased products (such as tempeh and miso) due to microorganism-induced hydrolysis of their respective glycoside conjugates into aglycones, whereas most nonfermented soybased products (such as tofu, soymilk, soy flour, etc.) largely contain mixtures of their malonyl-, acetyl-, and nonconjugated $\beta$-glycosides [9]. All glycosides are biologically inactive and poorly absorbed from the intestinal tract because of their hydrophilicity and larger molecular size [10]. Hydrolysis of the glycosidic bond using $\beta$-glucosidases is therefore necessary to release the biologically active aglycones which are rapidly absorbed across the intestinal wall $[7,10$, 11]. The $\beta$-glucosidases involved in this step are found in the small intestinal brush border (lactase phloridzin hydrolase $([\mathrm{LPH}]))[12]$ and within enterocytes (cytosolic $\beta$-glucosidases) [13]. Nonetheless, isoflavone glycosides that are not absorbed in the small intestine will pass through to the colon, where bacterial $\beta$-glycosidases can hydrolyze them and additional absorption can be exerted [10, 14-16]. Several groups of colonic bacteria including Lactobacillus spp., Bacteroides spp., and Bifidobacterium spp. possess $\beta$ glucosidase activity and have been demonstrated to be very effective deglycosylators [14].

Prebiotics such as fructooligosaccharides (FOSs) are poorly digested in the human small intestine but are fermented in the colon by the resident microflora [17]. FOS is also found to be fermented in vitro only by a specific range of microflora that include most species of bifidobacteria $[18,19]$. The oral administration of FOS for 7 days has been reported to significantly increase fecal bifidobacteria counts in healthy volunteers [20]. As aforementioned, this increased number of colonic bifidobacteria theoretically leads to an enhancement of bacterial $\beta$-glucosidase activity in the colon. It is possible that supplementation with FOS could increase the isoflavone absorption through enhancement of bacterial $\beta$-glucosidase activity [15]. Uehara et al. have shown that plasma and urine levels of genistein and daidzein are higher in the rat fed with FOS than the control rat [21]. In addition, an additive effect of dietary isoflavones and FOS has been demonstrated on the bone mineral density in ovariectomized mice [22]. However, the study of FOS on plasma isoflavone concentrations in human is still lacking. Therefore, the purpose of this study was to determine the influence of FOS on the pharmacokinetics of isoflavones in healthy, Thai postmenopausal women.

\section{Materials and Methods}

2.1. Study Design. The study was a fixed-sequence, twophase study with a washout period of at least one week. The study was approved by the Human Research Ethics Committee of the Faculty of Medicine, Chiang Mai University, and complied with the Helsinki Declaration.

2.2. Subjects. A total of 12 Thai postmenopausal women, age $>45 \mathrm{y}$, postmenopausal status $>1 \mathrm{y}$ (since the last sponta- neous menstrual bleeding), and serum follicle-stimulating hormone concentration $>30 \mathrm{IU} / \mathrm{L}$, were enrolled in this study. The body mass index (BMI) of subjects was within $18-25 \mathrm{~kg} / \mathrm{m}^{2}$. All had to be in good health based on medical history, physical examination, and routine blood tests including complete blood count, blood urea nitrogen, creatinine, and liver function tests. Exclusion criteria were history of malignancy, chronic renal, liver, cardiovascular, pulmonary or breast disease, a history of substance abuse or addiction, recent cigarette smoking, regular consumption of $>2$ alcoholic drinks/d, regular use of over-the-counter or prescription medication ( $>1$ dose/wk), use of antibiotics and laxatives within the previous 4 weeks, and intake of nutritional supplements (containing vitamins, minerals, fibers, FOS, isoflavones, other prebiotics or probiotics) within the previous 2 weeks.

2.3. Soy Preparation and FOS. The soy preparation used in this study was a commercial instant soy beverage D in 1 (manufactured by T.C. Pharmaceutical, Co., Ltd., Thailand), prepared by mixing 1 sachet $(36 \mathrm{~g})$ of soy powder with $300 \mathrm{~mL}$ of hot water. The mean isoflavone contents of daidzin and genistin were $11.24 \pm 2.84$ and $23.82 \pm 1.03 \mathrm{mg} /$ serving, respectively. The amounts of daidzein and genistein were negligible. FOS used in this study was Meioligo granule $2.5 \mathrm{~g} /$ sachets, manufactured by Meiji Seika Kaisha, Ltd., Tokyo, Japan.

2.4. Dosage and Drug Administration. Subjects were admitted to the Clinical Pharmacology Unit of the Faculty of Medicine, Chiang Mai University, at 6:30 AM after an overnight fast of at least $8 \mathrm{~h}$. Each received a single oral dose of $300 \mathrm{~mL}$ soy beverage $\left(\mathrm{D}_{0}\right)$ (single ISO phase). The subjects were instructed to remain upright and fast for $2 \mathrm{~h}$ after administration of the soy beverage. Water and lunch were served at $2 \mathrm{~h}$ and $6 \mathrm{~h}$ after dosing, respectively. Blood samples were collected at specific time points (see the following). Following blood sample collection at $12 \mathrm{~h}$ after the soy dose, the subjects were discharged from the Clinical Pharmacology Unit and were asked to come back again on the next day for blood sample collections at $24 \mathrm{~h}$ and $32 \mathrm{~h}$ after the initial dose. After a washout period of at least 1 week, subjects received oral doses of FOS, $5 \mathrm{~g}$ each time, twice a day (after breakfast and dinner) for 14 days $\left(\mathrm{D}_{-14}-\mathrm{D}_{-1}\right)$, followed by a single oral dose of $300 \mathrm{~mL}$ soy beverage on the next day $\left(\mathrm{D}_{0}\right)$ (continuous FOS/single ISO phase). Administrations of soy beverage and blood sample collections were performed in the same manner as in the former phase. An identical food and beverage containing no isoflavones were served during both phases. Subjects were required to refrain from drinking some beverages (e.g., soy milk, alcohol and caffeine containing beverages, etc.).

2.5. Blood Samples Collection. Venous blood samples $(7 \mathrm{~mL}$ each) for determination of plasma isoflavones were collected before the dose, and then at $0.5,1,2,4,6,8,10,12,24$, and $32 \mathrm{~h}$ after administration of the soy beverage. Samples were obtained from the forearm by venipuncture through 
an indwelling intravenous catheter and collected in a heparinized vacutainer. The blood collecting tubes were centrifuged at 1,200 rpm for $15 \mathrm{~min}$ and the plasma was separated and frozen at $-80^{\circ} \mathrm{C}$ for later analysis.

\subsection{Determination of Plasma Concentrations of Isoflavones}

2.6.1. Sample Preparation. The sample preparation and determination of isoflavone concentrations in plasma were modified from the method described by Teekachunhatean et al. [23]. An aliquot of $250 \mu \mathrm{L}$ of plasma was transferred to a $1.5 \mathrm{~mL}$ plastic vial and treated with $0.15 \mathrm{~mL}$ of a mixture of $\beta$-glucuronidase/sulfatase from Helix pomatia (Sigma G0876) to hydrolyze glucuronide and sulfate conjugates of genistein and daidzein. The enzyme mixture was made up freshly and contained $0.1 \mathrm{~g}$ ascorbic acid in $10 \mathrm{~mL}$ of $0.1 \mathrm{M}$ sodium acetate buffer, $0.01 \mathrm{~g}$ ethylenediaminetetraacetic acid (EDTA), and $250 \mu \mathrm{L}$ of Helix pomatia. The tubes containing the enzyme mixture were capped and heated overnight in a water bath $\left(15-18 \mathrm{~h}, 37^{\circ} \mathrm{C}\right)$ and then were allowed to cool to room temperature.

After enzymatic hydrolysis, plasma samples were spiked with $10 \mu \mathrm{L}$ of internal standard (IS, $50,000 \mathrm{ng} / \mathrm{mL}$ fluorescein in $80 \%$ methanol) and then deproteinated by mixing the plasma sample with $1,000 \mu \mathrm{L}$ of $1 \%$ acetic acid in acetonitrile, vortex mixing for $30 \mathrm{sec}$, and centrifuged at $14,000 \mathrm{rpm}$ for $10 \mathrm{~min}$, respectively. An aliquot of the supernatant was removed and evaporated to vacuum dried for $3 \mathrm{~h}$ at $60^{\circ} \mathrm{C}$. The residue was dissolved in $50 \mu \mathrm{L}$ of mobile phase $\mathrm{B}$, and $5 \mu \mathrm{L}$ of the sample was injected into the HPLC system. Chromatogram of isoflavone-free plasma is shown in Figure 1(a), whereas chromatogram of plasma containing 2,400 ng/mL of daidzein and genistein as well as $10,000 \mathrm{ng} / \mathrm{mL}$ of IS is presented in Figure 1(b).

2.6.2. High-Performance Liquid Chromatography (HPLC) Conditions. The assay of isoflavones was modified from the HPLC method and conditions previously described by Teekachunhatean et al. [23]. The samples were eluted on a C18 column (Inertsil, $150 \mathrm{~mm} \times 4$.6 I.D., $5 \mu \mathrm{m}$, GL Science, Tokyo, Japan) with a C18 guard column (Inertsil ODS-3, $10 \mathrm{~mm} \times 4.0$ I.D., $5 \mu \mathrm{m}$, GL Science, Tokyo, Japan). The chromatography condition consisted of two mobile phases. Mobile phase A was $55 \mathrm{mM}$ ammonium acetate in deionized water/acetonitrile/methanol $(250: 45: 45, \mathrm{v} / \mathrm{v} / \mathrm{v})$. Mobile phase B was $55 \mathrm{mM}$ ammonium acetate in deionized water/acetonitrile/methanol $(250: 255: 220, \mathrm{v} / \mathrm{v} / \mathrm{v})$. Both mobile phases contained $29 \mu \mathrm{L}$ of perchloric acid and $250 \mu \mathrm{L}$ of $1.44 \mathrm{mM}$ sodium dodecyl sulfate. Separation was performed at $25^{\circ} \mathrm{C}$. A gradient elution of $90 \%$ A with $10 \% \mathrm{~B}$ for $3.5 \mathrm{~min}, 50 \%$ A with $50 \% \mathrm{~B}$ at $3.5-6.5 \mathrm{~min}$, $30 \%$ A with $70 \%$ B at $6.5-9.2$, and $5 \%$ A with $95 \%$ B at 9.2-12.8 min was scheduled. The flow rate of mobile was maintained at $1 \mathrm{~mL} / \mathrm{min}$, and the analyses were detected by UV absorption at $259 \mathrm{~nm}$. The isoflavone contents of samples were determined by using a calibration curve of peak height ratios of isoflavones and IS versus respective isoflavone concentrations $(37.5,75,150,300,600,1,200$, and
$2,400 \mathrm{ng} / \mathrm{mL}$ ) with the use of linear regression. The linear regression analysis of peak height ratios of isoflavones versus isoflavone concentrations consistently yielded coefficients of determinant $\left(r^{2}\right)$ of 0.997 or better.

For intraday validation, 5 samples from each of 3 quality control (QC) samples $(112.5,1,100,2,200 \mathrm{ng} / \mathrm{mL})$ were evaluated with a single calibration curve. For interday validation, 5 sets of the 3 different concentrations of QC samples (112.5, $1,100,2,200 \mathrm{ng} / \mathrm{mL}$ ) were studied on 5 independent days with concurrent 5 standard calibration curves. The precision was reported as the percentage of coefficient of variation (\% $\mathrm{CV}$ ) which was calculated as follows:

$$
\% \mathrm{CV}=\frac{\mathrm{SD}}{\bar{X}} \times 100
$$

where SD was standard deviation and $\bar{X}$ was mean value of isoflavone concentration in plasma.

The deviation was expressed as the percentage of inaccuracy calculated by the following equation:

\% Deviation

$$
\begin{aligned}
& =\frac{(\text { Measured concentration }- \text { Spiked concentration })}{\text { Spiked concentration }} \\
& \times 100 \text {. }
\end{aligned}
$$

For the determination of daidzein concentrations in plasma, the \% CV of intraday precision for the 3 QC samples was $2.65 \%, 1.36 \%$, and $1.29 \%$, respectively, whereas that of interday precision was $6.04 \%, 1.10 \%$, and $3.05 \%$, respectively. The \% deviation of intraday assay for the 3 QC samples was $-5.49 \%,-1.32 \%$, and $-1.07 \%$, respectively, whereas that of interday precision was $-2.40 \%, 0.61 \%$, and $3.23 \%$, respectively.

For the determination of genistein concentrations in plasma, \% CV of intraday precision for the 3 QC samples was $4.48 \%, 1.71 \%$, and $1.30 \%$, respectively, whereas $\%$ CV of interday precision was $2.57 \%, 1.83 \%$, and $3.34 \%$, respectively. The \% deviation of intraday assay for the $3 \mathrm{QC}$ samples was $4.48 \%, 1.71 \%$, and $1.30 \%$, respectively, whereas those of interday precision was $2.52 \%,-0.40 \%$, and $2.46 \%$, respectively.

The mean recovery of daidzein and genistein from the determination procedure was $94.69 \pm 3.10 \%$ and $92.54 \pm$ $2.97 \%$, respectively.

\subsection{Data Analysis and Statistical Methods}

2.7.1. Pharmacokinetic Parameters. Maximal plasma concentration $\left(C_{\max }, \mathrm{ng} / \mathrm{mL}\right)$ and time to reach peak concentration $\left(T_{\max }, \mathrm{h}\right)$ were obtained directly by visual inspection of each subject's plasma concentration-time profile. The area under the plasma concentration-time curve from time 0 $32 \mathrm{~h}$ and $0-\infty \mathrm{h}\left(\mathrm{AUC}_{0-32}\right.$ and $\left.\mathrm{AUC}_{0-\infty}, \mathrm{ng} \cdot \mathrm{h} / \mathrm{mL}\right)$ as well as half-life $\left(t_{1 / 2}, \mathrm{~h}\right)$ was determined by noncompartmental analysis. The slope of the terminal log-linear portion of the concentration-time profile was determined by least-squares 


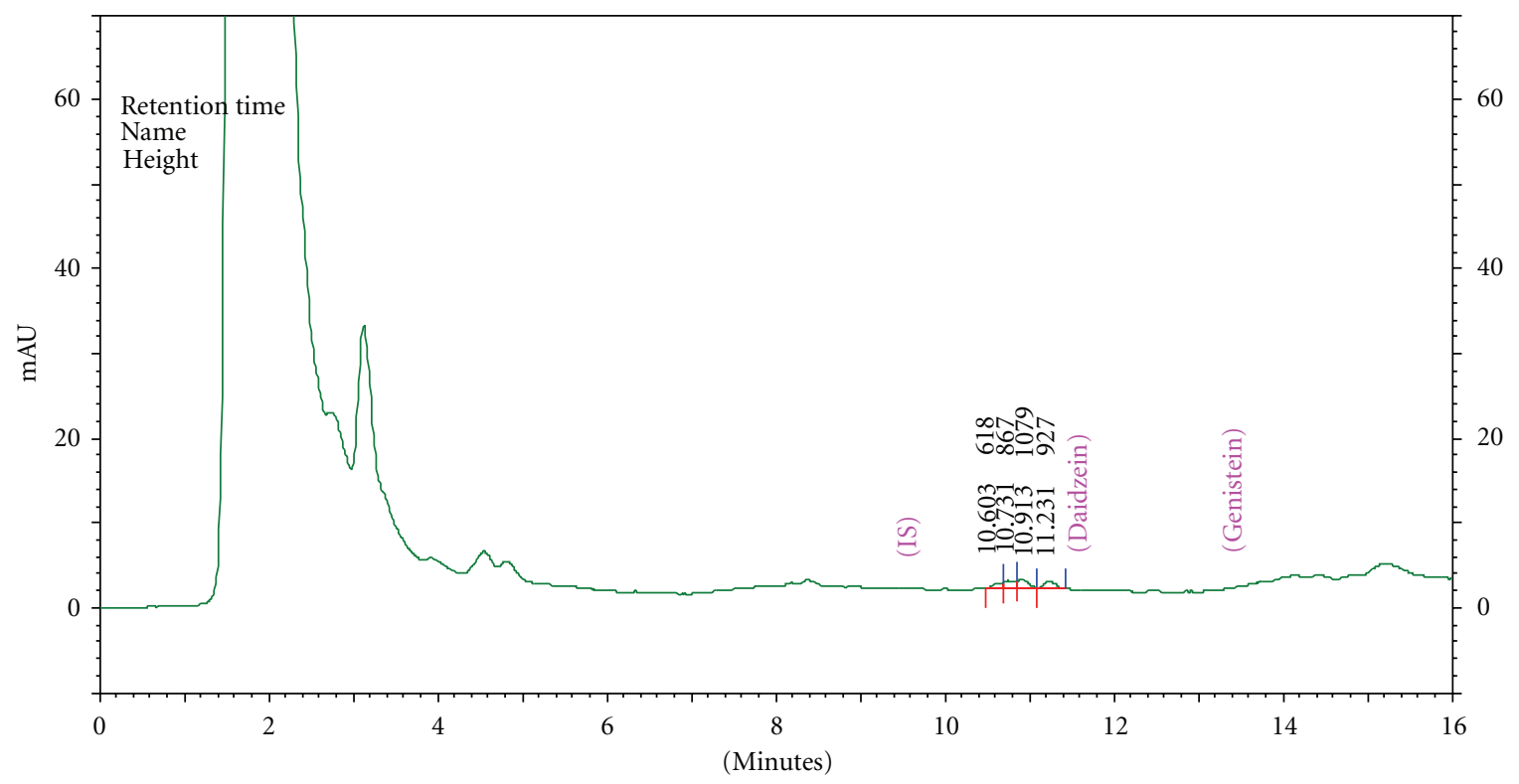

(a)

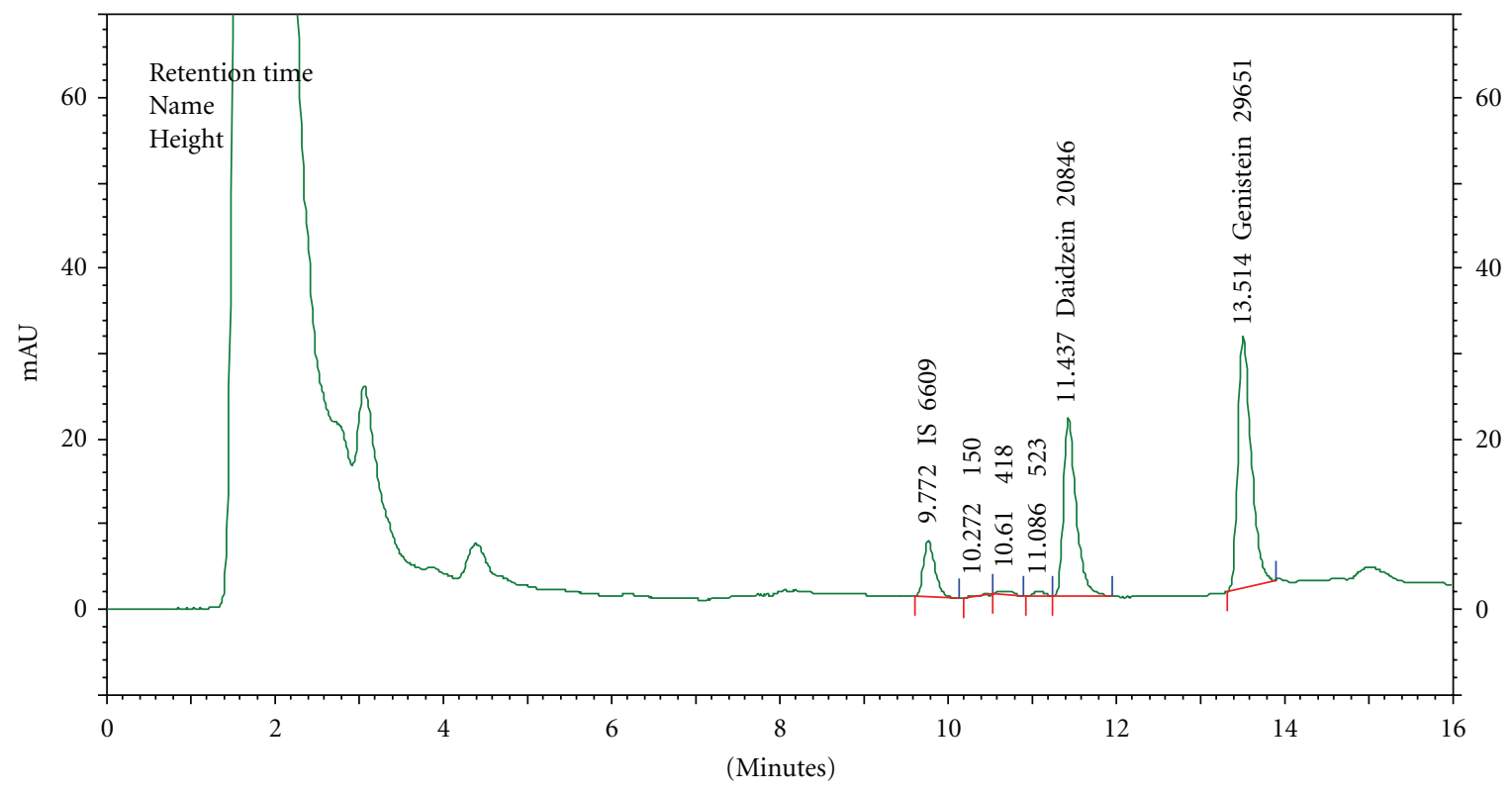

1: $259 \mathrm{~nm}, 8 \mathrm{~nm}$
validation
cal curve $1-107$

(b)

FIGURE 1: (a) Chromatogram of isoflavone-free plasma. (b) Chromatogram of plasma sample containing 2,400 ng/mL of daidzein $(k=$ $11.437 \mathrm{~min})$ and genistein $(k=13.514 \mathrm{~min})$ as well as $10,000 \mathrm{ng} / \mathrm{mL}$ of IS $(k=9.772 \mathrm{~min})$.

regression analysis and was used as the elimination rate constant $\left(k_{e}\right)$. The elimination half-life was calculated as $0.693 / k_{e}$. The AUC from time zero to the last quantifiable point $\left(\mathrm{AUC}_{0-32}\right)$ was calculated using the trapezoidal rule.
Extrapolated AUC from time $t$ to infinity $\left(\mathrm{AUC}_{t-\infty}\right)$ was determined as $\mathrm{Ct} / k_{e}$. Total $\mathrm{AUC}_{0-\infty}$ was the sum of $\mathrm{AUC}_{0-32}+$ $\mathrm{AUC}_{32-\infty}$. The calculation was performed by using the TopFit software version 2.0 for PC. 
TABLE 1: The demographic characteristics of 12 subjects enrolled in this study.

\begin{tabular}{lccccc}
\hline $\begin{array}{l}\text { Subject } \\
\text { No. }\end{array}$ & $\begin{array}{c}\text { Age } \\
(\mathrm{y})\end{array}$ & $\begin{array}{c}\text { Weight } \\
(\mathrm{kg})\end{array}$ & $\begin{array}{c}\text { Height } \\
(\mathrm{m})\end{array}$ & $\begin{array}{c}\text { BMI } \\
\left(\mathrm{kg} / \mathrm{m}^{2}\right)\end{array}$ & $\begin{array}{c}\text { FSH } \\
(\mathrm{mIU} / \mathrm{mL})\end{array}$ \\
\hline 1 & 56 & 57.00 & 1.52 & 24.67 & 41.56 \\
2 & 58 & 48.50 & 1.47 & 22.44 & 73.46 \\
3 & 64 & 59.00 & 1.60 & 23.05 & 61.71 \\
4 & 62 & 55.00 & 1.51 & 24.12 & 60.78 \\
5 & 50 & 58.50 & 1.65 & 21.49 & 90.77 \\
6 & 55 & 53.00 & 1.52 & 22.44 & 86.68 \\
7 & 54 & 59.00 & 1.54 & 24.88 & 79.67 \\
8 & 55 & 56.00 & 1.53 & 23.92 & 90.55 \\
9 & 53 & 48.50 & 1.52 & 20.99 & 125.84 \\
10 & 46 & 54.00 & 1.55 & 22.48 & 30.53 \\
11 & 67 & 42.00 & 1.46 & 19.70 & 80.14 \\
12 & 54 & 51.00 & 1.47 & 23.60 & 57.87 \\
\hline Mean & 56.17 & 53.46 & 1.53 & 22.81 & 73.30 \\
\hline SD & 5.87 & 5.18 & 0.05 & 1.55 & 25.22 \\
\hline
\end{tabular}

2.7.2. Statistical Analysis. The pharmacokinetic parameters were presented as mean $\pm \mathrm{SD}$. The differences in the mean values of pharmacokinetic parameters obtained from both phases were compared by using the Wilcoxon's signed-rank test. A $P$ value $<0.05$ was considered significant.

\section{Results}

The demographic characteristics of 12 female subjects enrolled in the study are shown in Table 1. All subjects completed the study.

The mean plasma concentration-time curves of daidzein and genistein from 12 subjects receiving single ISO versus continuous FOS/single ISO are shown in Figures 2 and 3, respectively. The plasma concentration-time profiles of daidzein and genistein in both phases showed wide intraindividual and interindividual variation but were typically biphasic in every individual regardless of the regimens taken (data not shown). The first and second peak concentrations of daidzein and genistein obtained from both phases were approximately reached at $2-4 \mathrm{~h}$ and $6-8 \mathrm{~h}$, respectively. The second peak concentrations of both isoflavones were always higher than the first peak.

The pharmacokinetic parameters of daidzein and genistein $\left(C_{\max }, \mathrm{AUC}_{0-32}, \mathrm{AUC}_{0-\infty}, T_{\max }, t_{1 / 2}\right)$ after oral administration of soy preparation in both phases were determined and are shown in Table 2. Of all pharmacokinetic parameters, it was shown that $\mathrm{C}_{\max }, \mathrm{AUC}_{0-32}$, and $\mathrm{AUC}_{0-\infty}$ of genistein as well as $\mathrm{AUC}_{0-32}$ of daidzein obtained from continuous FOS/single ISO phase were significantly higher than those of single ISO phase. For genistein, the mean $C_{\max }$ and AUC obtained from continuous FOS/single ISO phase were about $40 \%$ and $25 \%-30 \%$ and significantly higher comparing to those of "single ISO" phase. However, the mean $\mathrm{AUC}_{0-32}$ of daidzein obtained from "continuous FOS/single ISO" phase was only slightly $(\sim 15 \%)$ but statistically higher than that

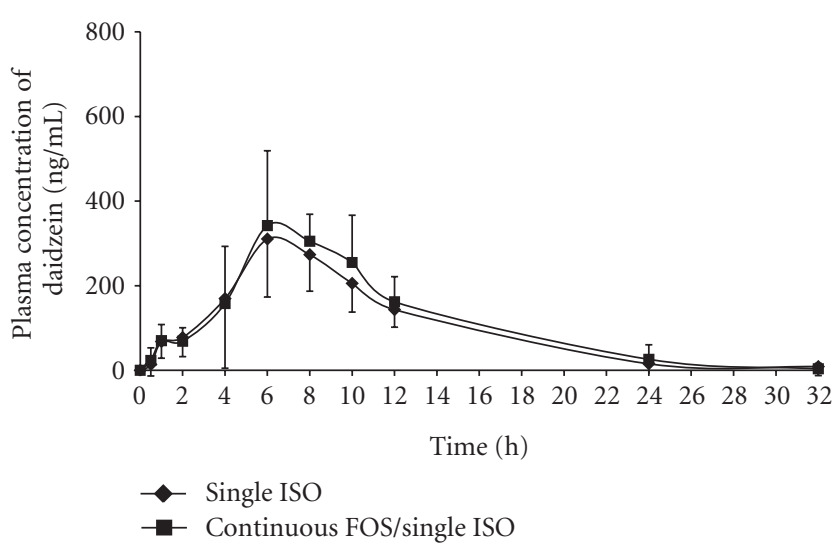

FIGURE 2: Mean plasma daidzein concentration-time curves from 12 subjects receiving a single oral administration of soy beverage (single ISO phase) and continuous oral administrations of FOS followed by a single oral administration of soy beverage (continuous FOS/single ISO phase).

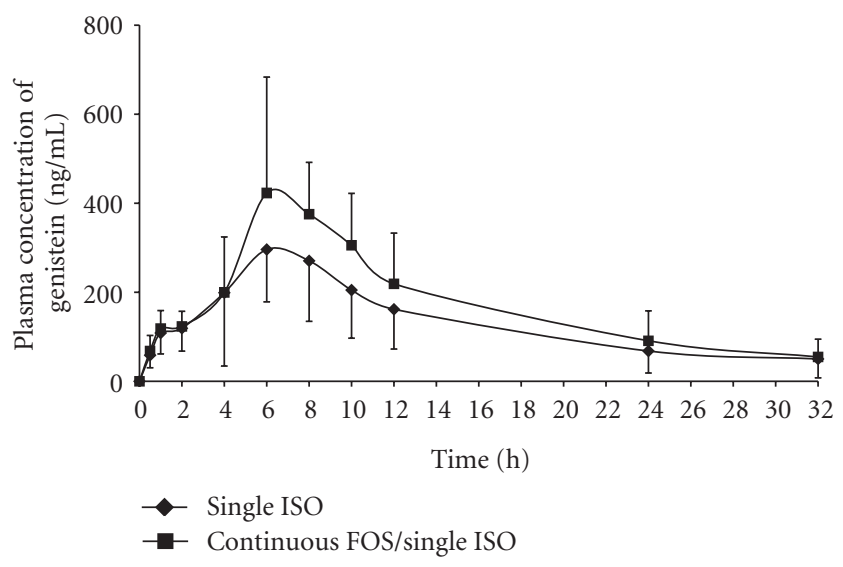

FIGURE 3: Mean plasma genistein concentration-time curves from 12 subjects receiving a single oral administration of soy beverage (single ISO phase) and continuous oral administrations of FOS followed by a single oral administration of soy beverage (continuous FOS/single ISO phase).

of "single ISO" phase. It is worth noting that significant increases in $\mathrm{AUC}_{0-32}$ of both aglycones in "continuous FOS/single ISO" phase were consistent with the higher second peaks of the plasma concentration-time curves, whereas the first peaks obtained from both phases were exactly the same.

Data from "continuous FOS/single ISO" phase revealed that 4 out of 12 postmenopausal subjects demonstrated only minimal increases in $\mathrm{AUC}_{0-32}(1 \%-10 \%$ increment) of daidzein, but 7 out of 12 demonstrated remarkable increases in $\mathrm{AUC}_{0-32}$ (>10\% increment). Similarly, 3 out of 12 demonstrated only minimal increases in $\mathrm{AUC}_{0-32}$ of genistein, and 8 out of 12 demonstrated remarkable increases in $\mathrm{AUC}_{0-32}$. Additionally, one subject demonstrated a decrease in $\mathrm{AUC}_{0-32}$ of both daidzein $(-33 \%)$ and genistein $(-16 \%)$. 
TABLE 2: Pharmacokinetic parameters of daidzein and genistein obtained from 12 subjects receiving a single oral administration of soy beverage (single ISO phase) and continuous oral administrations of FOS following by a single oral administration of soy beverage (continuous FOS/single ISO phase).

\begin{tabular}{lcccc}
\hline Pharmacokinetic parameters & \multicolumn{2}{c}{ Daidzein } & \multicolumn{2}{c}{ Genistein } \\
& Single ISO & Continuous FOS/single ISO & Single ISO & Continuous FOS/single ISO \\
\hline $\mathrm{C}_{\max }(\mathrm{ng} / \mathrm{mL})$ & $363.33 \pm 116.67$ & $412.90 \pm 100.61$ & $347.01 \pm 143.42$ & $489.65 \pm 187.15^{*}$ \\
$\mathrm{AUC}_{0-32}(\mathrm{ng} \cdot \mathrm{h} \cdot \mathrm{mL})$ & $2753.71 \pm 1227.64$ & $3177.32 \pm 1474.55^{*}$ & $4096.66 \pm 2050.96$ & $5354.39 \pm 2650.16^{*}$ \\
$\mathrm{AUC}_{0-\infty}(\mathrm{ng} \cdot \mathrm{h} \cdot \mathrm{mL})$ & $3399.35 \pm 1261.82$ & $3741.85 \pm 1421.28$ & $5075.62 \pm 2787.77$ & $6321.17 \pm 3047.63^{*}$ \\
$T_{\max }(\mathrm{h})$ & $6.67 \pm 1.78$ & $6.67 \pm 1.78$ & $6.00+1.91$ & $6.67 \pm 1.56$ \\
$t_{1 / 2}(\mathrm{~h})$ & $4.65 \pm 2.46$ & $5.27 \pm 2.50$ & $9.61 \pm 4.45$ & $10.01 \pm 4.17$ \\
\hline
\end{tabular}

Data represents mean \pm SD. ${ }^{*} P<0.05$ versus single ISO phase.

\section{Discussion}

This is the first study to investigate the influence of FOS on pharmacokinetics of isoflavones in healthy postmenopausal women. Twelve subjects in this study were enrolled in accordance to the number of subjects in the previous study demonstrating that supplementation with prebiotic inulin significantly increases the extent of isoflavone absorption [24].

Despite the mixtures of malonyl-, acetyl-, and $\beta$-glycoside conjugates of daidzein and genistein are the major isoflavones found in most nonfermented soy-based products [16], both malonyl- and acetyl-conjugates can readily be hydrolyzed to their respective more heat-stable $\beta$-glycoside conjugates during exposure to processing temperature [9]. Hence this is the reason why daidzein and genistein were demonstrated to be the major forms of isoflavones in the instant soy beverage used in this study. The soy preparation containing the predominant isoflavone glycosides (daidzin and genistin) rather than aglycone forms was chosen because it has been hypothesized that FOS increases isoflavone bioavailability via stimulation of gut microflora ability to hydrolyze isoflavone glycosides to their respective more readily absorbable aglycones. Additionally, soy beverage was considered the formulation of choice rather than other commercially available soy extract capsules because the $\beta$ glycoside conjugates dissolved in soy beverage are ready to be hydrolyzed and further absorbed without the necessity of determining their disintegration and dissolution profiles, which are important confounding factors during absorption.

Although daidzin and genistin in the ingested soy beverage need to be hydrolyzed by the intestinal or gut microflora $\beta$-glucosidases to release free aglycones prior to absorption, the absorbed aglycones are further predominantly metabolized to their $\beta$-glucuronide conjugates, and to lesser amounts also as sulfate conjugates, in the intestine and/or liver [25]. Therefore, the glucuronide and sulfate conjugates are the major metabolites of isoflavones found in systemic circulation. Treating plasma samples with a mixture of $\beta$-glucuronidase/sulfatase before determination of plasma isoflavone concentrations in this study resulted in enzymatic hydrolysis of glucuronide and sulfate conjugates to aglycones (daidzein and genistein). Thus, plasma concentrations of the respective aglycones were determined rather than their glucuronide and sulfate conjugates [23].

The mean $T_{\max }$ of daidzein $(6.67 \pm 1.78 \mathrm{~h})$ and genistein $(6.00 \pm 1.91 \mathrm{~h})$ obtained from single ISO phase of this study was quite shorter than that of $8-11 \mathrm{~h}$ after ingestion of isoflavone conjugates reported in other studies [26, 27]. This discrepancy might be as a result of such factors as ethnic background, soy preparations used, intestinal microflora, and dietary habits. However, the $T_{\max }$ reported here was comparable to that of our previous studies in Thai postmenopausal women $[23,28]$. Additionally, the mean $t_{1 / 2}$ of daidzein and genistein was $4.65 \pm 2.46 \mathrm{~h}$ and $9.61 \pm 4.45 \mathrm{~h}$, respectively, which are in agreement with those of 3-9h for daidzein and 8-11 h for genistein after the intake of soy foods or pure isoflavone glycosides [29, 30].

In this study, a typical biphasic pattern appeared in both phases for all individuals, as well as in mean plasma concentrations-time curves of postmenopausal subjects. This finding is consistent from those reported elsewhere $[26,29,31]$. Plasma concentrations of isoflavones could be detected as early as $30 \mathrm{~min}$, and the first peak was attained at approximately $2-4 \mathrm{~h}$ after soy intake. It has been reported that this early detection of plasma levels and the presentation of the first peak correspond to the hydrolysis of $\beta$-glycoside conjugates and initial absorption of aglycones readily occurring in the duodenum and proximal jejunum $[26,29,32,33]$. Additionally, the second peak attained at $6-8 \mathrm{~h}$ corresponds to an ability of gut microflora to cleave $\beta$-glycosides to their respective aglycones before the latter can be absorbed mainly in the large intestine $[10,14-$ 16]. The large intestine is known to be the location where resident microflora are present in the largest numbers and consequently play the most crucial role for the uptake of isoflavones [33]. The significant delay of the second peak after the radical reduction of the gut flora, achieved by mechanical bowel preparation in combination with oral antibiotic treatment, emphasizes further evidence that this peak corresponds to colonic bacterial $\beta$-glucosidase activity [33].

In the "continuous FOS/single ISO" phase of this study, the significant increases in $C_{\max }$ (of genistein) and AUC (of both daidzein and genistein), compared to those of "single ISO" phase, correlated to the higher second peaks 
of the plasma concentration-time curves. These findings suggest that pretreatment of FOS for 14 days might efficiently lead to stimulation of microflora growth especially in the colon, resulting in enhancement of microflora $\beta$-glucosidases activity, and hence increased oral absorption of both aglycones. These are consistent with the previous study, which demonstrated that oral administration of FOS significantly increases the absorption of both aglycones in rats, especially during 6-48 h after isoflavone intake [21]. Similarly, study in healthy postmenopausal women has shown that plasma concentrations of both aglycones are significantly higher after the oral consumption of isoflavones plus prebiotic inulin for 21 days, compared to those of without inulin [24].

The present investigation revealed that the majority of study subjects demonstrated remarkable increases in AUC ( $>10 \%$ increment) of both aglycones, whereas the minority (approximately $40 \%$ of participants) showed minimal increases or even decrease in these parameters. This discrepancy reflects the different potential of FOS to stimulate gut microflora growth, which might be due mainly to variations in basal amount and type of resident microflora responsible for isoflavone conversion, as well as their ability to metabolize glycoside conjugates in the intestinal milieu of different individuals [34]. Although FOS seems likely to stimulate a limited range of microorganisms, especially bifidobacteria [18], it is speculated that the growth of other bacterial species in some individuals might be simultaneously stimulated, leading to an increase in the biotransformation of isoflavones to metabolites other than their respective aglycones; for example, daidzein can be further metabolized to equol and $O$-desmethylangolensin, and genistein to p-ethyl phenol [16]. Indeed, it appears that more than one bacterial species could be involved in the metabolic conversions of these aglycones [35]. On the other hand, it is still uncertain whether an increased amount of bifidobacteria under some specific circumstances could further metabolize daidzein and genistein to other constituents resulting in lower oral bioavailability of these aglycones in some individuals. Therefore, the correlation between changes in isoflavone bioavailability versus the fecal bacterial counts (including bifidobacteria and others) and/or $\beta$-glucosidase activity in the fecal contents after FOS supplement should be further investigated, to clarify the unanswered question of how FOS affects the bacterial growth especially in subjects whose isoflavone bioavailability cannot be increased or even decreased.

Interestingly, present and previous studies have unanimously demonstrated that FOS or inulin remarkably enhances the absorption of genistein, rather than daidzein both in rats [21], and in human subjects [24]. Since the combination of dietary FOS and isoflavones has been shown to correlate with increased plasma equol levels in rats [36, 37], and $30 \%$ of humans can metabolize daidzein to equol that is detectable in systemic circulation [15], it is hypothesized that enhancement of the conversion of daidzein to equol (and/or $O$-desmethylangolensin) from increased bacterial growth following FOS supplement could explain why it is less likely to increase the bioavailability of daidzein in comparison to genistein. Nonetheless, the role of bifidobacteria involving in the reductive pathway of daidzein toward equol is still controversial. Although the formation of equol in soymilk fermented with some strains of bifidobacteria has been reported [38], 22 strains of bifidobacteria fail to transform daidzein into equol under various experimental conditions in vitro. These findings exclude any role of bifidobacteria in the production of equol [39]. Thus, the determination of plasma concentrations of equol especially in subjects demonstrating lesser enhancement of isoflavone bioavailability after FOS supplement should be further studied. However, the presentation of biologically active equol in plasma after FOS supplement, if any, might be able to produce beneficial effects in postmenopausal women despite no significant increase in the bioavailability of daidzein, because equol possesses more estrogenic activity than daidzein and binds more strongly to the estrogen receptors [40]. It also has a longer half-life and superior antioxidant activity $[41,42]$.

Since the second peak of both isoflavones was demonstrated to be at approximately $2 \mathrm{~h}$ after lunch in most subjects enrolled in this study, this finding indicates that the $\beta$-glucuronide and sulfate conjugates excreted via bile during lunch time might be further deconjugated by the gut microflora and undergo enterohepatic recirculation as suggested by previous studies $[43,44]$. This additional absorption of isoflavones into systemic circulation possibly accounts for the second surge of plasma concentrations [29]. Whether stimulation of bacterial growth after FOS administration contributes to enhancement of gut microflora $\beta$ glucuronidase activity, and hence increase in enterohepatic recirculation during the second peak, is still equivocal and warrants further investigation.

In the present study, continuous administration of FOS could increase AUC of daidzein and genistein by approximately $15 \%$ and $30 \%$, respectively, whereas other studies showed that doubling the oral dose of soy nuts from 20 to $40 \mathrm{~g}$ yields increments of AUC by approximately $80 \%$ and $40 \%$, respectively [30]. Therefore, increased oral ingestion of isoflavones or soy foods seems to be a simple but more effective and less costly means to enhance oral isoflavone bioavailability in comparison to coadministration of fixed-dose isoflavones plus FOS. Nonetheless, FOS has been found to increase intestinal absorption of calcium in rats [45] and humans [46, 47], presumably by stimulating growth of resident microflora such as bifidobacteria. In addition, soy foods combined with a prebiotic significantly improve the lipid profiles in hyperlipidemic adults, possibly via an increase in colonic microbial biotransformation of isoflavones and/or specific short-chain fatty acids [48]. Therefore, coadministration of isoflavones plus FOS might play some advantageous role in certain circumstances, such as in postmenopausal women with coexisting osteoporosis and/or dyslipidemia.

The major limitations of this study were listed as follows. Firstly, since the carry-over effect of FOS on enhancement of bacterial growth especially in vivo is not well understood, the study was therefore designed as fixed-sequence rather than a randomized-sequence cross-over study in order to avoid any possible residual effect of FOS. Secondly, a further limitation 
is the lack of quantification of fecal bacterial counts, $\beta$ glucosidase activity in the fecal content, and plasma levels of equol.

\section{Conclusion}

Continuous oral administrations of FOS followed by a single oral administration of soy beverage caused significant increases in $C_{\max }, \mathrm{AUC}_{0-32}$, and $\mathrm{AUC}_{0-\infty}$ of genistein and $\mathrm{AUC}_{0-32}$ of daidzein, compared to those obtained following a single oral dose of soy beverage alone.

\section{Acknowledgment}

This work was supported by the Faculty of Medicine, Chiang Mai University, Thailand. All authors do not have a direct financial relation with the commercial identities mentioned in the paper and have no conflict of interests.

\section{References}

[1] K. C. Nichols, L. Schenkel, and H. Benson, "17 $\beta$-Estradiol for postmenopausal estrogen replacement therapy," Obstetrical and Gynecological Survey, vol. 39, no. 4, pp. 230-245, 1984.

[2] M. P. Cust, K. F. Gangar, T. C. Hillard, and M. I. Whitehead, "A risk-benefit assessment of estrogen therapy in postmenopausal women," Drug Safety, vol. 5, no. 5, pp. 345-358, 1990.

[3] C. John, "Treatment for menopausal symptoms," The American Journal of Medicine, vol. 34, pp. 31-37, 2006.

[4] M. Hickey, S. R. Davis, and D. W. Sturdee, "Treatment of menopausal symptoms: what shall we do now?" Lancet, vol. 366, no. 9483, pp. 409-421, 2005.

[5] J. E. Rossouw, G. L. Anderson, R. L. Prentice, A. Z. LaCroix, C. Kooperberg, and M. L. Stefanick, "Writing Group for the Women's Health Initiative Investigators. Risks and benefits of estrogen plus progestin in healthy postmenopausal women: principal results from the Women's Health Initiative randomized controlled trial," Journal of the American Medical Association, vol. 288, pp. 321-333, 2002.

[6] M. P. Warren, B. Shortle, and J. E. Dominguez, "Use of alternative therapies in menopause," Best Practice and Research: Clinical Obstetrics and Gynaecology, vol. 16, no. 3, pp. 411448, 2002.

[7] K. D. R. Setchell, "Phytoestrogens: the biochemistry, physiology, and implications for human health of soy isoflavones," American Journal of Clinical Nutrition, vol. 68, no. 6, 1998.

[8] A. Cassidy, "Potential risks and benefits of phytoestrogenrich diets," International Journal for Vitamin and Nutrition Research, vol. 73, no. 2, pp. 120-126, 2003.

[9] P. A. Murphy, K. Barua, and C. C. Hauck, "Solvent extraction selection in the determination of isoflavones in soy foods," Journal of Chromatography B, vol. 777, no. 1-2, pp. 129-138, 2002.

[10] X. Xu, K. S. Harris, H. J. Wang, P. A. Murphy, and S. Hendrich, "Bioavailability of soybean isoflavones depends upon gut microflora in women," Journal of Nutrition, vol. 125, no. 9, pp. 2307-2315, 1995.

[11] A. Scalbert and G. Williamson, "Dietary intake and bioavailability of polyphenols," Journal of Nutrition, vol. 130, no. 8, pp. S2073-S2085, 2000.

[12] A. J. Day, F. J. Cañada, J. C. Díaz et al., "Dietary flavonoid and isoflavone glycosides are hydrolysed by the lactase site of lactase phlorizin hydrolase," FEBS Letters, vol. 468, no. 2-3, pp. 166-170, 2000.

[13] A. J. Day, M. S. Dupont, S. Ridley et al., "Deglycosylation of flavonoid and isoflavonoid glycosides by human small intestine and liver $\beta$-glucosidase activity," FEBS Letters, vol. 436, no. 1, pp. 71-75, 1998.

[14] T. E. Steer, I. T. Johnson, J. M. Gee, and G. R. Gibson, "Metabolism of the soyabean isoflavone glycoside genistin in vitro by human gut bacteria and the effect of prebiotics," British Journal of Nutrition, vol. 90, no. 3, pp. 635-642, 2003.

[15] C. Atkinson, C. L. Frankenfeld, and J. W. Lampe, "Gut bacterial metabolism of the soy isoflavone daidzein: exploring the relevance to human health," Experimental Biology and Medicine, vol. 230, no. 3, pp. 155-170, 2005.

[16] C. R. Cederroth and S. Nef, "Soy, phytoestrogens and metabolism: a review," Molecular and Cellular Endocrinology, vol. 304, no. 1-2, pp. 30-42, 2009.

[17] C. Molis, B. Flourié, F. Ouarne et al., "Digestion, excretion, and energy value of fructooligosaccharides in healthy humans," American Journal of Clinical Nutrition, vol. 64, no. 3, pp. 324-328, 1996.

[18] G. R. Gibson and X. Wang, "Enrichment of bifidobacteria from human gut contents by oligofructose using continuous culture," FEMS Microbiology Letters, vol. 118, no. 1-2, pp. 121128, 1994.

[19] T. Tokunaga, Y. Nakada, T. Yasuhito, M. Hirayama, and H. Hidaka, "Effects of fructooligosaccharides intake on the intestinal microflora and defecation in healthy volunteers," Bifidus, vol. 6, pp. 143-150, 1993.

[20] Y. Bouhnik, K. Vahedi, L. Achour et al., "Short-chain fructooligosaccharide administration dose-dependently increases fecal bifidobacteria in healthy humans," Journal of Nutrition, vol. 129, no. 1, pp. 113-116, 1999.

[21] M. Uehara, A. Ohta, K. Sakai, K. Suzuki, S. Watanabe, and H. Adlercreutz, "Dietary fructooligosaccharides modify intestinal bioavailability of a single dose of genistein and daidzein and affect their urinary excretion and kinetics in blood of rats," Journal of Nutrition, vol. 131, no. 3, pp. 787-795, 2001.

[22] L. Devareddy, D. A. Khalil, K. Korlagunta, S. Hooshmand, D. D. Bellmer, and B. H. Arjmandi, "The effects of fructooligosaccharides in combination with soy protein on bone in osteopenic ovariectomized rats," Menopause, vol. 13, no. 4, pp. 692-699, 2006.

[23] S. Teekachunhatean, P. Pongnad, N. Rojanasthein, M. Manorot, and C. Sangdee, "Effects of vitamin D plus calcium supplements on pharmacokinetics of isoflavones in thai postmenopausal women," Evidence-based Complementary and Alternative Medicine, vol. 2011, Article ID 895471, 7 pages, 2011.

[24] C. Piazza, M. G. Privitera, B. Melilli et al., "Influence of inulin on plasma isoflavone concentrations in healthy postmenopausal women," American Journal of Clinical Nutrition, vol. 86, no. 3, pp. 775-780, 2007.

[25] L. Coward, M. Kirk, N. Albin, and S. Barnes, "Analysis of plasma isoflavones by reversed-phase HPLC-multiple reaction ion monitoring-mass spectrometry," Clinica Chimica Acta, vol. 247, no. 1-2, pp. 121-142, 1996.

[26] K. D. R. Setchell, N. M. Brown, P. Desai et al., "Bioavailability of pure isoflavones in healthy humans and analysis of commercial soy isoflavone supplements," Journal of Nutrition, vol. 131, no. 4, supplement, pp. 1362S-1375S, 2001.

[27] R. A. King and D. B. Bursill, "Plasma and urinary kinetics of the isoflavones daidzein and genistein after a single soy meal in 
humans," American Journal of Clinical Nutrition, vol. 67, no. 5, pp. 867-872, 1998.

[28] E. Anupongsanugool, S. Teekachunhatean, N. Rojanasthien, S. Pongsatha, and C. Sangdee, "Pharmacokinetics of isoflavones, daidzein and genistein, after ingestion of soy beverage compared with soy extract capsules in postmenopausal Thai women," BMC Clinical Pharmacology, vol. 5, article no. 2, 2005.

[29] S. Watanabe, M. Yamaguchi, T. Sobue et al., "Pharmacokinetics of soybean isoflavones in plasma, urine and feces of men after ingestion of $60 \mathrm{~g}$ baked soybean powder (kinako)," Journal of Nutrition, vol. 128, no. 10, pp. 1710-1715, 1998.

[30] K. D. R. Setchell, N. Maynard Brown, P. B. Desai et al., "Bioavailability, disposition, and dose-response effects of soy isoflavones when consumed by healthy women at physiologically typical dietary intakes," Journal of Nutrition, vol. 133, no. 4, pp. 1027-1035, 2003.

[31] S. Vergne, K. Titier, V. Bernard et al., "Bioavailability and urinary excretion of isoflavones in humans: effects of soy-based supplements formulation and equol production," Journal of Pharmaceutical and Biomedical Analysis, vol. 43, no. 4, pp. 1488-1494, 2007.

[32] M. Richelle, S. Pridmore-Merten, S. Bodenstab, M. Enslen, and E. A. Offord, "Hydrolysis of isoflavone glycosides to aglycones by $\beta$-glycosidase does not alter plasma and urine isoflavone pharmacokinetics in postmenopausal women," Journal of Nutrition, vol. 132, no. 9, pp. 2587-2592, 2002.

[33] A. A. Franke, L. J. Custer, and S. A. Hundahl, "Determinants for urinary and plasma isoflavones in humans after soy intake," Nutrition and Cancer, vol. 50, no. 2, pp. 141-154, 2004.

[34] F. Rafii, C. Davis, M. Park, T. M. Heinze, and R. D. Beger, "Variations in metabolism of the soy isoflavonoid daidzein by human intestinal microfloras from different individuals," Archives of Microbiology, vol. 180, no. 1, pp. 11-16, 2003.

[35] H. G. Hur, J. O. Lay, R. D. Beger, J. P. Freeman, and F. Rafii, "Isolation of human intestinal bacteria metabolizing the natural isoflavone glycosides daidzin and genistin," Archives of Microbiology, vol. 174, no. 6, pp. 422-428, 2000.

[36] A. Ohta, M. Uehara, K. Sakai et al., "A combination of dietary fructooligosaccharides and isoflavone conjugates increases femoral bone mineral density and equol production in ovariectomized mice," Journal of Nutrition, vol. 132, no. 7, pp. 2048-2054, 2002.

[37] J. Mathey, C. Puel, S. Kati-Coulibaly et al., "Fructooligosaccharides maximize bone-sparing effects of soy isoflavoneenriched diet in the ovariectomized rat," Calcified Tissue International, vol. 75, no. 2, pp. 169-179, 2004.

[38] D. Tsangalis, J. F. Ashton, A. E. J. Mcgill, and N. P. Shah, "Enzymic transformation of isoflavone phytoestrogens in soymilk by $\beta$-glucosidase-producing bifidobacteria," Journal of Food Science, vol. 67, no. 8, pp. 3104-3113, 2002.

[39] S. Raimondi, L. Roncaglia, M. De Lucia et al., "Bioconversion of soy isoflavones daidzin and daidzein by Bifidobacterium strains," Applied Microbiology and Biotechnology, vol. 81, no. 5, pp. 943-950, 2009.

[40] V. Breinholt, A. Hossaini, G. W. Svendsen, C. Brouwer, and S. E. Nielsen, "Estrogenic activity of flavonoids in mice. The importance of estrogen receptor distribution, metabolism and bioavailability," Food and Chemical Toxicology, vol. 38, no. 7, pp. 555-564, 2000.

[41] K. D. R. Setchell and A. Cassidy, "Dietary isoflavones: biological effects and relevance to human health," Journal of Nutrition, vol. 129, no. 3, pp. 758S-767S, 1999.
[42] X. L. Wang, H. J. Kim, S. I. Kang, S. I. Kim, and H. G. Hur, "Production of phytoestrogen S-equol from daidzein in mixed culture of two anaerobic bacteria," Archives of Microbiology, vol. 187, no. 2, pp. 155-160, 2007.

[43] J. Sfakianos, L. Coward, M. Kirk, and S. Barnes, "Intestinal uptake and biliary excretion of the isoflavone genistein in rats," Journal of Nutrition, vol. 127, no. 7, pp. 1260-1268, 1997.

[44] J. Chen, H. Lin, and M. Hu, "Metabolism of flavonoids via enteric recycling: role of intestinal disposition," Journal of Pharmacology and Experimental Therapeutics, vol. 304, no. 3, pp. 1228-1235, 2003.

[45] A. Ohta, M. Ohtsuki, S. Baba, T. Adachi, T. Sakata, and E. Sakaguchi, "Calcium and magnesium absorption from the colon and rectum are increased in rats fed fructooligosaccharides," Journal of Nutrition, vol. 125, no. 9, pp. 2417-2424, 1995.

[46] C. Coudray, J. Bellanger, C. Castiglia-Delavaud, C. Rémésy, M. Vermorel, and Y. Rayssignuier, "Effect of soluble or partly soluble dietary fibres supplementation on absorption and balance of calcium, magnesium, iron and zinc in healthy young men," European Journal of Clinical Nutrition, vol. 51, no. 6, pp. 375-380, 1997.

[47] E. G. H. M. Van Den Heuvel, T. Muys, W. Van Dokkum, and G. Schaafsma, "Oligofructose stimulates calcium absorption in adolescents," American Journal of Clinical Nutrition, vol. 69, no. 3, pp. 544-548, 1999.

[48] J. M. W. Wong, C. W. C. Kendall, R. De Souza et al., "The effect on the blood lipid profile of soy foods combined with a prebiotic: a randomized controlled trial," Metabolism: Clinical and Experimental, vol. 59, no. 9, pp. 1331-1340, 2010. 


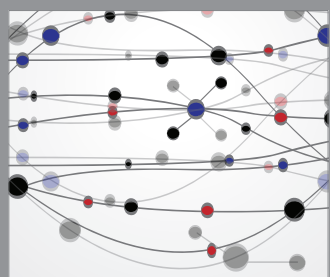

The Scientific World Journal
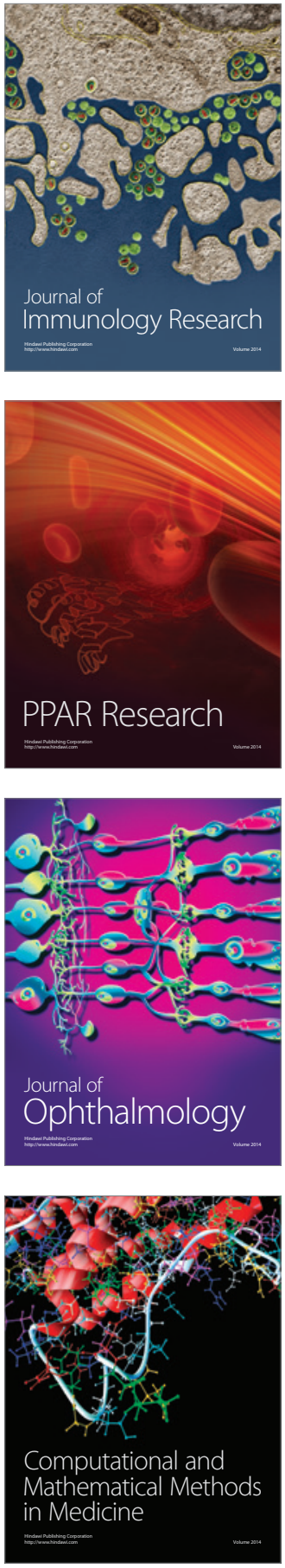

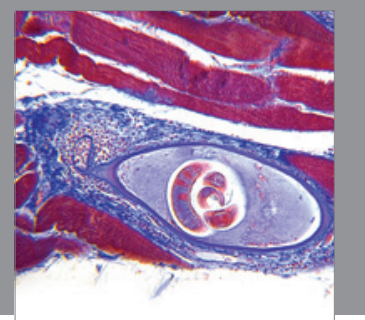

Gastroenterology

Research and Practice
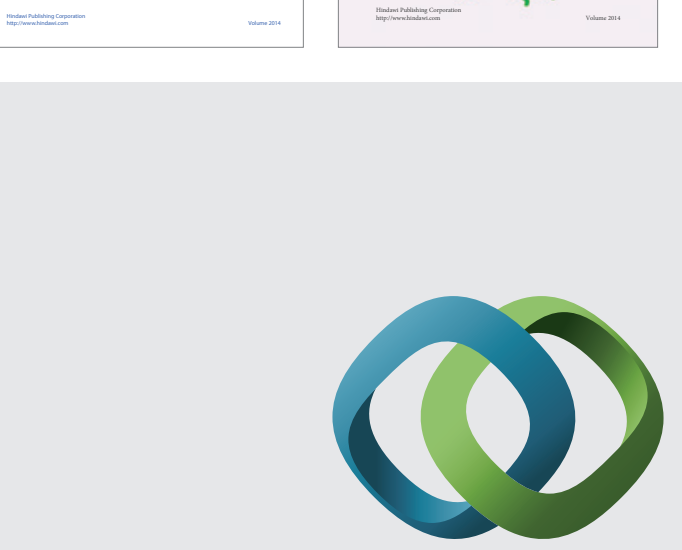

\section{Hindawi}

Submit your manuscripts at

http://www.hindawi.com
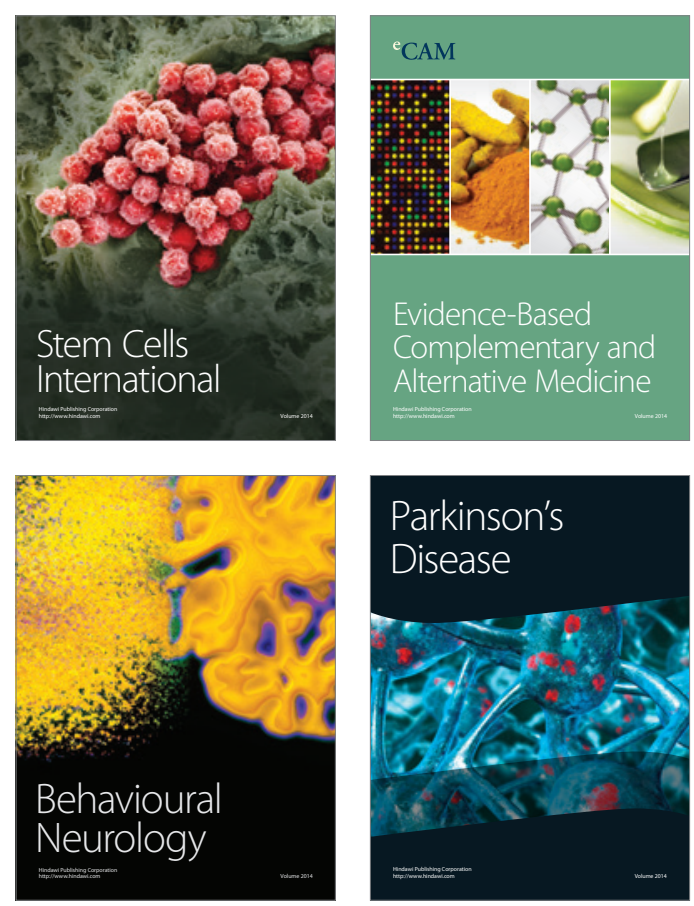

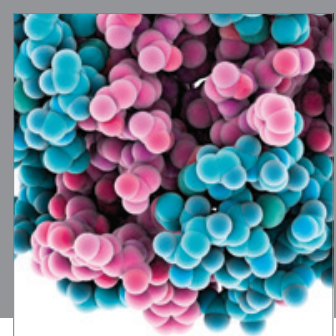

Journal of
Diabetes Research

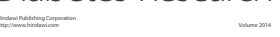

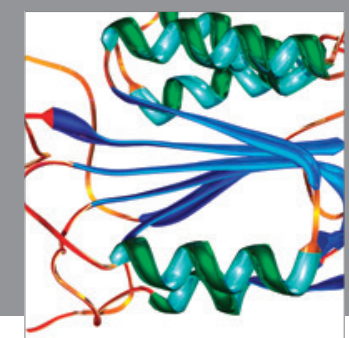

Disease Markers
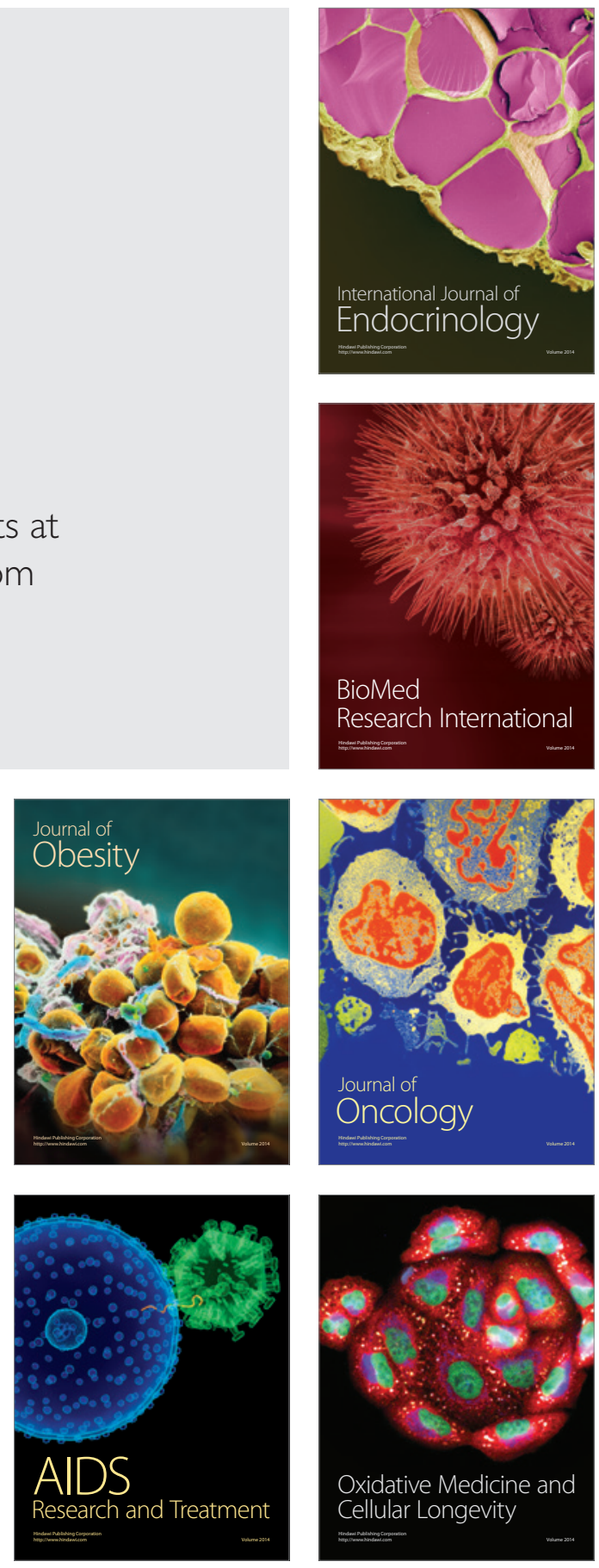